\title{
Meeting Report: Consensus Recommendations for a Research Agenda in Exercise in Solid Organ Transplantation
}

\author{
S. Mathur ${ }^{1}$, T. Janaudis-Ferreira ${ }^{2}$, \\ L. Wickerson ${ }^{1,3}$, L. G. Singer ${ }^{3,4}$, J. Patcai ${ }^{2,4}$, \\ D. Rozenberg ${ }^{4}$, T. Blydt-Hansen ${ }^{5}$, \\ E. L. Hartmann ${ }^{6}$, M. Haykowsky ${ }^{7,8}$, D. Helm³, \\ K. High ${ }^{9}$, N. Howes ${ }^{10}$, B. M. Kamath ${ }^{11,12}$, \\ L. Lands ${ }^{13,14}$, S. Marzolini ${ }^{15}$ and \\ C. Sonnenday ${ }^{16,17}$
}

\footnotetext{
${ }^{1}$ Department of Physical Therapy, University of Toronto, Toronto, ON, Canada

${ }^{2}$ Sunnybrook Research Institute, Sunnybrook Health Sciences Centre - St John's Rehab Program, Toronto, ON, Canada

${ }^{3}$ Toronto Lung Transplant Program - University Health Network, Toronto, ON, Canada

${ }^{4}$ Department of Medicine, University of Toronto, Toronto, ON, Canada

${ }^{5}$ Department of Pediatrics and Child Health, Division of Nephrology, University of Manitoba, Winnipeg, MB, Canada

${ }^{6}$ Piedmont Transplant Institute, Atlanta, GA

${ }^{7}$ Faculty of Rehabilitation Medicine, University of Alberta, Edmonton, AB, Canada

${ }^{8}$ Mazankowski Alberta Heart Institute, Edmonton, $A B$, Canada

${ }^{9}$ Department of Internal Medicine, Wake Forest School of Medicine, Winston-Salem, NC

${ }^{10}$ Multi-Organ Transplant Program, London Health

Sciences Centre, London, ON, Canada

${ }^{11}$ Department of Paediatrics, University of Toronto, Toronto, ON, Canada

${ }^{12}$ Division of Gastroenterology, Hepatology and Nutrition, Hospital for Sick Children, Toronto, ON, Canada

${ }^{13}$ Department of Pediatrics, Division of Pediatric

Respiratory Medicine, McGill University Health Centre, Montréal, OC, Canada

${ }^{14}$ Quebec Lung Transplant Program, Centre Hospitalier de l'Université de Montréal, Montréal, QC, Canada

${ }^{15}$ Toronto Rehabilitation Institute - University Health

Network, Cardiovascular Prevention and Rehabilitation

Program, Toronto, ON, Canada

${ }^{16}$ Department of Surgery, University of Michigan, Ann Arbor, MI

${ }^{17}$ Department of Health Management \& Policy, University of Michigan, Ann Arbor, MI

* Corresponding author: Sunita Mathur,

sunita.mathur@utoronto.ca

Sunita Mathur and Tania Janaudis-Ferreira are co-first authors.
}

\begin{abstract}
With improved survival rates in solid organ transplantation there has been an increased focus on long-term outcomes following transplant, including physical function, health-related quality-of-life and cardiovascular mortality. Exercise training has the potential to affect these outcomes, however, research on the optimal timing, type, dose of exercise, mode of delivery and relevant outcomes is limited. This article provides a summary of a 2-day meeting held in April 2013 (Toronto, Canada) in which a multi-disciplinary group of clinicians, researchers, administrators and patient representatives engaged in knowledge exchange and discussion of key issues in exercise in solid organ transplant (SOT). The outcomes from the meeting were the development of top research priorities and a research agenda for exercise in SOT, which included the need for larger scale, multi-center intervention studies, development of standardized outcomes for physical function and surrogate measures for clinical trials, examining novel modes of exercise delivery and novel outcomes from exercise training studies such as immunity, infection, cognition and economic outcomes. The development and dissemination of "expert consensus guidelines," synthesizing both the best available evidence and expert opinion was prioritized as a key step toward improving program delivery.
\end{abstract}

Abbreviations: $\mathrm{QOL}$, quality-of-life; RCT, randomized controlled trial; SOT, solid organ transplant; $\mathrm{VO}_{2}$ peak, peak oxygen consumption

Received 03 December 2013, revised 16 May 2014 and accepted for publication 31 May 2014

\section{Introduction}

Solid organ transplantation is a life-saving intervention for people with end-stage heart, lung, kidney or liver disease. With considerable advances in organ preservation, surgical techniques and immunosuppressive therapy, short-term survival following solid organ transplant (SOT) has greatly improved. Specifically over the last decade, the 1 year patient survival for heart, lung, kidney and liver transplants from deceased donors have improved from approximately $85 \%$ to over $90 \%$ on average, across transplant types (1). As a result of improved graft survival and reduced deaths from infection/rejection there has been a shift in focus toward sustaining improvements in quality-of-life (OOL), 


\section{Mathur et al}

reducing morbidity from cardiovascular disease and improving long-term survival in transplant recipients (2). Current evidence suggests that greater physical function in transplant candidates and recipients is associated with lower pretransplant mortality and improved posttransplant outcomes. For example, low physical activity levels have been strongly associated with increased risk for cardiovascular and all-cause mortality in renal transplant recipients (2). In lung and liver transplant recipients, higher pretransplant exercise capacity has been associated with lower pretransplant mortality $(3,4)$, shorter hospital stay $(5)$ and increased short-term survival posttransplant (6). Furthermore, better perceptions of physical function (using the Short Form-36 self-report questionnaire) in renal transplant recipients have been associated with a lower hazard for hospitalization and death posttransplant (7).

Exercise training, including aerobic, resistance or combined training, has been shown to improve physical function and QOL in SOT recipients (8). Structured exercise training in transplant recipients also has the potential to reduce cardiovascular risk factors, such as hypertension (9), percent body fat (10) and aerobic fitness (8). Although several randomized controlled trials (RCTs) have been conducted in SOT recipients, questions still remain regarding the optimal timing, frequency and dose of exercise prescription in this population. Furthermore, there are differing opinions on whether transplant recipients are unique in their needs, responses and adaptations to exercise training compared to others living with chronic disease. The long-term effects of exercise and physical activity on important clinical outcomes from transplant such as survival, risk of rejection and sustained improvements in QOL, as well as the cost-effectiveness of exercise training interventions have not been systematically studied. Therefore, the benefit of exercise training specific to the SOT population is not fully understood.

These areas of debate provided the foundation for the Exercise in Solid Organ Transplant Meeting, which was held in Toronto, Canada, April 18-20th 2013. This 2-day meeting was the first of its kind in North America, and brought together researchers, clinicians and stakeholders with expertise in rehabilitation/exercise across different SOTs. The goal of the meeting was to identify common research opportunities and areas of inquiry across SOT and put a unified effort toward producing high impact research in exercise and rehabilitation for transplant recipients, and consequently improve clinical practice. This meeting report summarizes the current evidence for exercise limitation and exercise training in SOT and presents the research agenda developed at the meeting.

\section{Current Evidence}

\section{Exercise limitation}

It is well accepted that heart and lung transplant candidates have limited pretransplant exercise capacity due to their primary organ failure, therefore, the majority of exercise studies have been focused on these transplant populations (8). However, people with chronic kidney or liver disease also demonstrate limitations in exercise capacity pretransplant, often due to secondary consequences of disuse, such as muscle weakness (11) rather than a consequence of their primary disease process. Furthermore, there is a consistent observation across SOT recipients that aerobic capacity (or peak oxygen consumption [ $\mathrm{VO}_{2}$ peak]) is impaired preoperatively and remains below age-matched normative values posttransplant (11). The limitation in $\mathrm{VO}_{2}$ peak appears to be related to peripheral muscle dysfunction (impaired muscle oxygen extraction and utilization) rather than central factors such as cardiovascular or respiratory limitations, even in heart and lung transplant recipients (11). Furthermore, peripheral adaptations have been observed as a result of exercise training in SOT recipients, such as improved blood lactate (12), mitochondrial function (13), muscle strength (14) and an increase in oxidative, type 1 muscle fibers (14).

Although each SOT has its own unique characteristics and challenges, many issues that affect exercise capacity and physical function are common across the transplant types (11). Pretransplant factors such as the physiological changes associated with severe chronic disease, deconditioning and nutritional depletion can affect exercise capacity and physical function in the transplant candidate. However, the goals of exercise training in the transplant candidate differ from chronic disease rehabilitation, since pretransplant rehabilitation is focused on maintaining physical function during a period of rapid decline in health and preparing for a complex surgery which is followed by a period of hospitalization and bedrest.

Following transplant, extended hospital and intensive care stay, prolonged sedentary time, immunosuppressant medications and episodes of organ rejection may all impact the transplant recipients' exercise tolerance and healthrelated $\mathrm{QOL}$. In the early posttransplant phase, the recipients are more complex and medically unstable than typical patients undergoing rehabilitation. A recent study showed that transplant recipients undergoing inpatient rehabilitation had 10 times higher rate of readmission to the acute hospital compared to other patient populations participating in rehabilitation (e.g. neurological, musculoskeletal) (15). The long-term limitations in exercise capacity posttransplant may be due to the effects of immunosuppressant medications such as corticosteroids, which have been associated with muscle myopathy (16) and calcineurin inhibitors, which have been shown to affect mitochondrial respiration and muscle regeneration/remodeling (17) in animal models. In the posttransplant phase, the exercise training goals are substantially different from the pretransplant phase and from chronic disease. Since transplant recipients experience major improvements in symptoms, function and $\mathrm{QOL}$, they can focus on gaining higher levels of physical function and re-engaging in societal roles (work, 


\section{Exercise in Solid Organ Transplantation}

leisure and family obligations). Exercise and physical activity in the posttransplant phase are part of a longterm commitment that may lead to sustained improvements in physical function, $\mathrm{QOL}$ and potentially improved survival.

Interestingly, the recommendation for rehabilitation following transplant differ based on organ type. Cardiac rehabilitation guidelines recommend rehabilitation as standard of care treatment following heart transplant (18). However, guidelines for pulmonary rehabilitation focus on individuals with stable disease or acute exacerbation, but do not include lung transplant candidates or recipients (19). Therefore, it remains unclear whether the unique needs of transplant candidates and recipients could benefit from specific rehabilitation and exercise training strategies.

\section{Evidence for exercise training in SOT}

It is well documented that SOT recipients have significant physical impairments and increased risk for cardiovascular disease and that exercise training has potential for both short- and long-term benefits in this population. Yet, there is a lack of high quality RCTs with long-term follow-up on exercise training for transplant recipients. In a recent systematic review examining the health benefits and risks associated with exercise following SOT (8) only 15 RCTs were identified across kidney $(n=2)$, liver $(n=1)$, heart $(n=9)$ and lung $(n=3)$ transplant populations; the majority of which were conducted in cardiac transplant. The limited number of studies, particularly in kidney and liver transplant populations, despite the higher number of these organ transplants done annually compared to heart and lung, is likely a reflection of the evidence-based guidelines in exercise training for people with cardiac and pulmonary disease $(18,19)$. Whereas, exercise training for people with chronic kidney or liver disease has not been established with the same level of evidence.

Table 1 shows the characteristics of the published exercise training programs and their effects on the main outcomes. Ultimately, stakeholders are interested in long-term outcomes such as survival, sustained improvement in QOL and cost-effectiveness. However, none of the existing RCTs measured these outcomes. The authors of the recent systematic review noted that $20 \%$ of these RCTs (three trials) were considered as having high risk of bias based on quality appraisal (8). A summary of the main findings of this systematic review is presented below.

Exercise capacity and muscle strength: The studies included in the meta-analysis showed an improvement in $\mathrm{VO}_{2}$ peak among recipients of cardiac (six studies) but not in kidney (one study), liver (two studies) or lung transplant (one study). Differences among the exercise training protocols may explain the conflicting results. However, when data from all the trials were pooled, there was a greater change in $\mathrm{VO}_{2}$ peak among transplant recipients who underwent training (standardized mean difference of $0.47 \mathrm{~mL} \cdot \mathrm{kg} \cdot \mathrm{min}^{-1}$ ). The clinical relevance of this change is not established in the transplant population; however, it is well below the threshold seen in cardiovascular disease, where a $3.5 \mathrm{~mL} \cdot \mathrm{kg} \cdot \mathrm{min}^{-1}$ increase in $\mathrm{VO}_{2}$ peak has been shown to be associated with an $8 \%$ to $14 \%$ improvement in survival (20).

Six studies included muscle strength as an outcome; of these, four studies included a strength training component and only one of these studies demonstrated a greater increase in muscle strength in lung transplant recipients after exercise training compared to a control group (9). Interestingly, of the two studies that did not include any strength training $(10,21)$, one also showed a statistically significant increase in muscle strength in kidney transplant recipients (21). This may have been due to the low level of conditioning of the subjects at baseline, leading to strength improvements even with aerobic training.

Cardiopulmonary variables: Three studies showed a decrease in systolic blood pressure $(9,22,23)$ and two studies showed significantly lower diastolic blood pressure in lung and heart recipients following training $(9,22)$.

Body composition: Percentage body fat has been shown to be reduced after exercise training in cardiac (24) and liver transplant recipients (10). Lumbar bone mineral density (BMD) has been shown to increase (compared to pretransplant values) in cardiac recipients after exercise training (25) while no increase has been observed in lung (26) or liver transplant recipients (10).

Health-related QOL: Six studies assessed QOL and there was evidence that exercise training improves $\mathrm{QOL}$ in cardiac transplant recipients $(27,28)$. There was limited evidence that exercise training improves $\mathrm{QOL}$ in lung $(9,29)$ or liver (10) transplant recipients.

Other outcomes: Only one RCT (9) has considered physical activity as an outcome and showed an improvement in physical activity levels in lung transplant recipients after training. This study also observed a trend of lower incidence of diabetes in the trained group (9).

\section{Meeting Process}

Three specific objectives were addressed at the meeting: (1) increase communication and collaboration of experts across different types of SOT to develop a wider research network; (2) identify areas of commonality and uniqueness in the current state of knowledge regarding exercise and physical function in SOT, in an effort to consolidate research efforts across transplant types; and (3) identify "high leverage" research questions in exercise-based rehabilitation for SOT. 


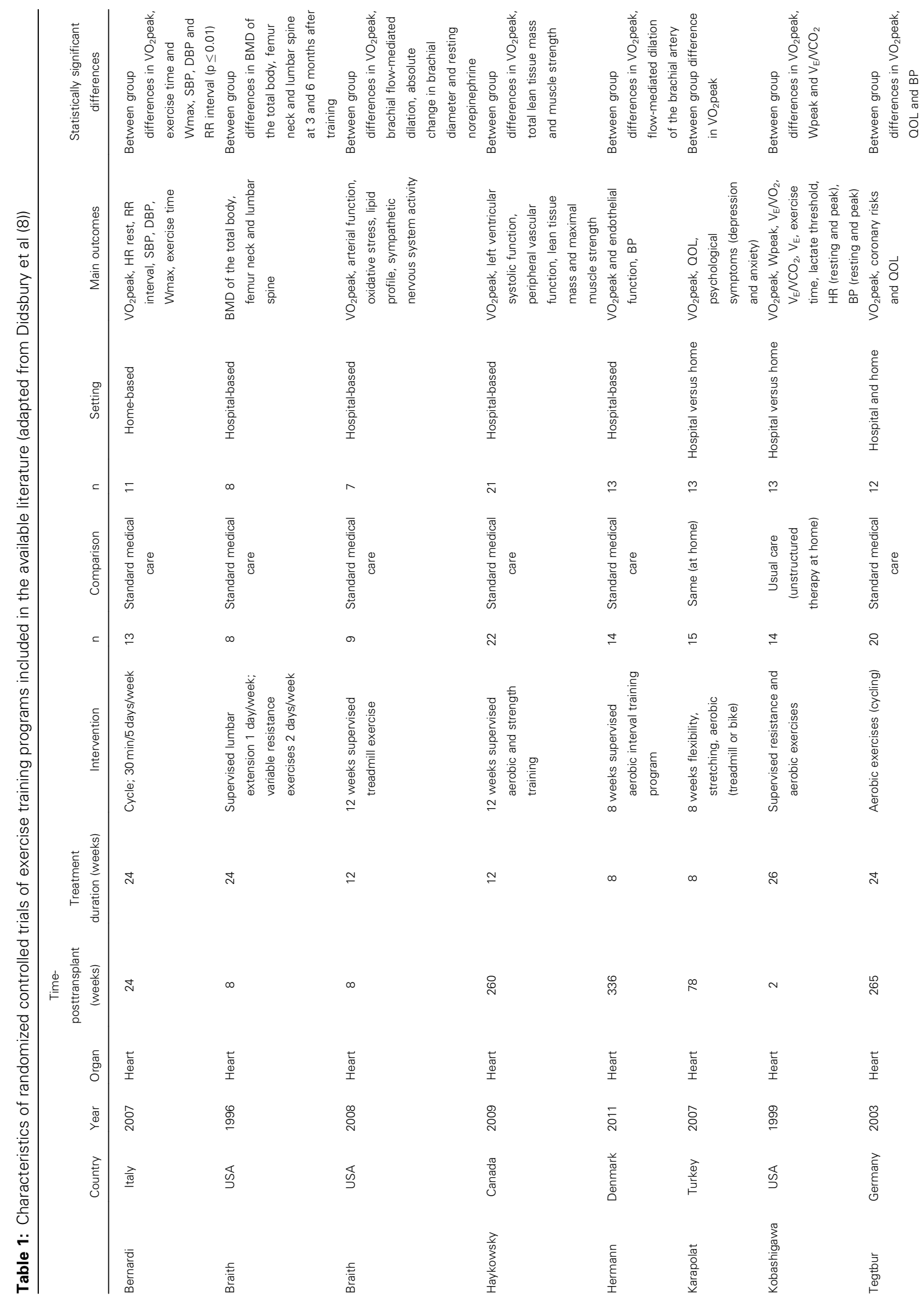




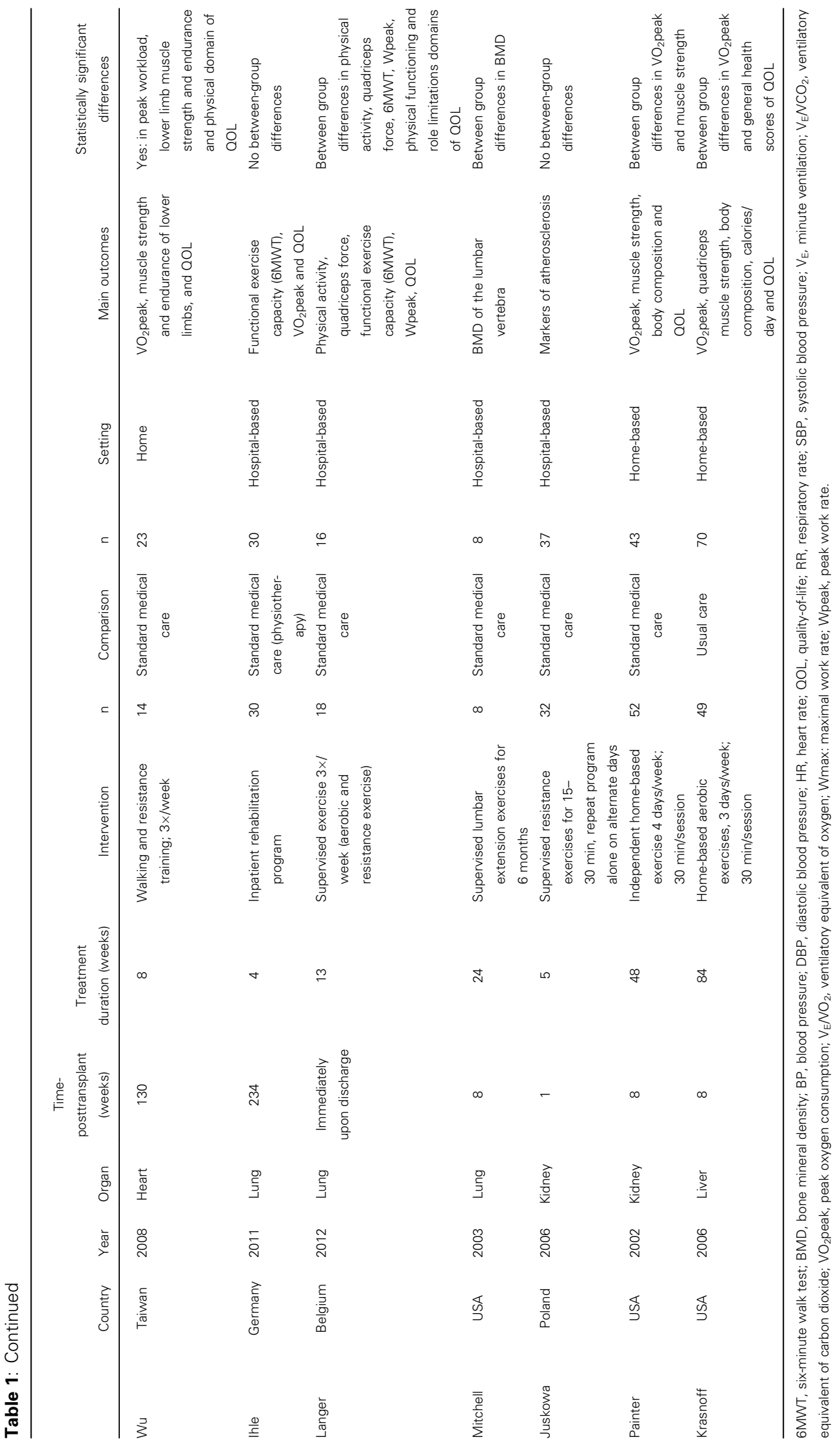




\section{Mathur et al}

The 2-day meeting was led by a group of five coinvestigators (SM, TJ-F, LW, LGS, JP) and 11 additional collaborators from across Canada and the United States. Prior to the meeting, an electronic survey was circulated to the invitees to stimulate ideas around the topic of exercise for SOT. A total of 60 participants attended the first day of the meeting including researchers, clinicians, health-care administrators and patient representatives. This portion of the meeting was a knowledge exchange where invited speakers presented the evidence for exercise training and exercise limitation in SOT and potential areas for future research (see Supporting Information for agenda). The remainder of the meeting was allocated to small group discussions leading to the development of a research agenda on exercise for SOT (see Supporting Information for discussion topics) and developing steps for future engagement of a collaborative research network. Participation on day 2 was limited to 23 individuals interested in this goal.

\section{Meeting Outcomes}

The ideas generated from the group discussions on topics related to exercise in SOT are summarized below. The top research priorities and the research agenda developed from the meeting are provided in Tables 2 and 3. A key priority was the dissemination of the current evidence for transplant rehabilitation and the identification for the need to conduct more high quality trials to allow the development of evidence-based guidelines. The other top priorities focused on the development of standard assessments, which could be collected in a large national database for transplant rehabilitation research and the identification of surrogate outcomes that could be applied in clinical trials of exercise interventions.

\section{Assessment and outcome measures for clinical trials in exercise}

The development of a large rehabilitation/physical function database for transplant centers and standardized procedures for outcomes was identified as a top priority to creating a national database for transplant research. Physical function measures that were proposed for a database included standardized physical function tests which typically consist of timed functional tasks such as walking a short distance or rising from a chair (e.g. Short Physical Performance Battery), physical activity question- naires which measure frequency and duration of engagement in structured exercise, sports, leisure time and household activities, self-reported activity of daily living scales (e.g. Barthel Index) and indices of physical frailty (e.g. slowness, exhaustion, weakness, weight loss, low physical activity). The need for measures to be validated in the transplant population was also discussed.

\section{Prediction of outcomes}

Although there is evidence that exercise capacity is associated with mortality on the waiting list in transplant candidates (4), there are a limited number of studies on the effects of pretransplant exercise capacity on posttransplant outcomes (7), indicating a highly relevant area of research. The identification of surrogate markers for hard clinical outcomes, such as survival and $\mathrm{QOL}$ was voiced during the discussions. For example, in the pretransplant phase it was suggested to study the predictive validity of physical function, physical activity and frailty as assessed by their associations with posttransplant survival and QOL.

\section{Standard interventions and approaches to exercise training}

Although RCTs in exercise were identified from the literature in all SOTs, there was a need to further understand and define the nature of the exercise intervention itself, with particular attention to the phase of transplant: pre-, early-post- and late-posttransplant interventions (Table 3). Exploring "dose-dependent" effects of exercise training and defining the key elements of an exercise program were discussed. The type of training (aerobic, resistance), intensity of training (traditional moderate-intensity versus high-intensity interval training), duration of the program and preexercise assessments are highly variable in the literature. Therefore, future studies need to clearly define the training variables and progression of exercise, in order to examine the "dose-response" relationship between exercise and health-related outcomes in transplant. The role of immunosuppressant medications in modulating the effect of exercise training adaptations was also a key issue that requires further study in transplant recipients. There was discussion on the need to examine late posttransplant care using a chronic disease management framework to ensure long-term adherence to exercise and physical activity and to promote selfmanagement.

Table 2: Top research priorities identified to address future research in exercise for solid organ transplant

\footnotetext{
1. Standards for assessment: what are the best tools to evaluate exercise capacity, function and frailty in transplant candidates and recipients? What are the measurement properties of these tools in disease-specific or multi-organ transplant populations?

2. Surrogate outcomes for clinical trials in exercise: which outcomes/measures of exercise capacity, function and frailty are associated with hard clinical outcomes such as quality-of-life and survival in the pre- and posttransplant phases?

3. Knowledge translation: dissemination of current evidence and identification of the gaps in evidence to relevant stakeholders (e.g. clinicians, health-care administrators, researchers, patients/families)
} 
Exercise in Solid Organ Transplantation

Table 3: High leverage research questions for exercise in solid organ transplant

Pretransplant
Assessment and
outcomes measures
Standard interventions/
approaches

Novel interventions/ approaches

Health-care economics

Early posttransplant ${ }^{1}$

Assessment/outcome measures

Standard interventions/ approaches

Novel interventions/ approaches

Health-care economics

Late posttransplant ${ }^{2}$

Assessment/outcome measures

Standard interventions/ approaches

Novel interventions/ approaches
What is the concurrent of pretransplant physical function measures [exercise capacity $\left(\mathrm{VO}_{2}\right.$ peak, six-minute walk test [6MWT]), muscle strength, physical activity (questionnaires, step counts, energy expenditure) and functional mobility (gait speed, sit to stand test)] as assessed by associations with clinical outcomes such as quality-of-life?

What is the predictive validity of pretransplant physical function measures as outlined above, as assessed by associations with posttransplant outcomes such as survival and quality-of-life?

What is the effect of pretransplant frailty on transplant outcomes in adult organ transplant groups?

Is there evidence of frailty in pediatric organ transplant candidates? What are the associations between frailty and transplant outcomes in the pediatric population?

Does exercise training in the pretransplant phase improve clinical outcomes such as mortality on the waiting list, length of hospital stay, discharge destination posttransplant or early posttransplant survival?

What is the optimal timing and duration of pretransplant rehabilitation?

Is there a measurable improvement in markers of exercise capacity (e.g. $6 \mathrm{MWT}, \mathrm{VO}_{2}$ peak, muscle strength) with pretransplant exercise training? Do these improvements translate to better quality-of-life during the waiting period?

Is the response to exercise training dependent on the pretransplant diagnosis (within and across organ groups) or other pretransplant factors (e.g. age, initial level of fitness, frailty)?

Can high-intensity interval training (HIIT) be safely applied in pretransplant candidates? Does interval training confer greater training benefits in terms of aerobic fitness in pretransplant candidates compared to conventional endurance training?

Can circuit training (combination of resistance and aerobic training) confer similar benefits as conventional aerobic training in pretransplant candidates with lower cardiovascular and respiratory demands during training?

Can resistance training (with or without nutritional intervention) improve sarcopenia in frail patients pretransplant?

Is pretransplant exercise training cost-effective? What is the impact on health-care utilization during the waiting period (physician and specialist visits, emergency room visits etc)?

Which functional measures in the early posttransplant phase are the best predictors/surrogate markers of survival and quality-of-life?

Does a structured, supervised rehabilitation program starting after hospital discharge, lead to a sustained improvement of quality-of-life?

Can a multi-organ transplant exercise program provide similar benefits in exercise outcomes (exercise capacity, quality-of-life) as a single-organ exercise program?

Can home-based or community-based exercise training confer similar benefits to traditional in-center programs in the early posttransplant phase?

Is there a cost-benefit to delivering a supervised exercise program to a multi-organ group compared to single-organ group in the early posttransplant period?

What are the best functional predictors/surrogate markers of long-term survival in solid organ transplant recipients (i.e. $\mathrm{VO}_{2}$ peak, muscle mass)?

Is greater functional capacity associated with greater participation outcomes, such as return to work?

Does exercise training and/or physical activity have an effect on long-term survival and quality-of-life in transplant recipients?

Do steroids have an effect on the muscle hypertrophic response and muscle regenerative capacity in response to structured resistance training in transplant recipients?

What is the role of calcineurin inhibition on preventing muscle recovery following transplantation? How do exercise-induced muscle changes differ between transplant recipients receiving low levels of calcineurin inhibition to those with higher doses?

Does a "trained athlete" transplant recipient have a better ability to adapt to exercise training than a typical transplant recipient? What factors predict the "athlete's" ability to reach higher levels of fitness (i.e. training volume, age, sex, immunosuppressant medications)?

Does long-term exercise training and/or physical activity affect risk of organ rejection, allograft survival, risk of opportunistic infection?

What is the effect of exercise training on cognition, particularly in older adult transplant recipients?

What is the effect of long-term exercise and lifestyle physical activity in development of obesity,

hypertension, diabetes and/or metabolic syndrome in adult and pediatric transplant recipients?

What factors affect long-term adherence to structured exercise or lifestyle physical activity in children and adults after transplant (e.g. self-efficacy for exercise, motivators and barriers)? 


\section{Mathur et al}

Is a pedometer-based walking program effective in improving long-term adherence to physical activity in transplant recipients?

Is a chronic disease framework an effective way to improve self-management after transplant?

Health-care economics

What are the most cost-effective ways to support exercise and physical activity in the long-term management of transplant recipients?

Does long-term adherence to exercise training result in lower health-care utilization and cost-benefit in the posttransplant period?

\footnotetext{
${ }^{1}$ Early posttransplant phase defined starting from the time of hospital discharge after transplantation up to 12 months posttransplant

${ }^{2}$ Late posttransplant phase defined as greater than 12 months posttransplant.
}

\section{Novel interventions and approaches to exercise training}

Studies on different modes of aerobic training such as interval (characterized by short periods of high-intensity exercise alternated with longer periods of lower intensity exercise) or circuit weight training (characterized by a series of weight lifting exercises completed with minimal rests to elicit gains in cardiovascular and musculoskeletal fitness) were identified as modes of training that could be applicable to certain sub-groups of patients but required further study. For example, interval training has been studied in a limited number of transplant patients but has promising results to date in heart transplant recipients (30). Also the role for resistance training combined with nutritional interventions to improve muscle strength and muscle mass, particularly in frail patients in the pretransplant phase, was identified as a future area of study.

There was discussion on issues surrounding single-organ versus multi-organ (referring to individuals with different types of SOTs rather than multiple transplanted organs, such as heart-lung or liver-pancreas) exercise studies. There was a general consensus that multi-organ transplant research could have several advantages over single-organ research such as access to a greater sample size and opportunities for sub-group analyses across transplant types. Although transplant candidates could participate in existing rehabilitation programs within their primary disease groups, the improvement in functional status posttransplant and the issues regarding immunosuppressant medications as well as infection and rejection, pose unique questions for all transplant recipients regardless of the primary organ disease. These similarities could justify a posttransplant rehabilitation strategy for all types of SOT recipients. It was also recognized that the level of evidence for exercise training differs between the organ types since greater emphasis has been placed in heart and lung transplant. Therefore, the starting point for exercise training studies may differ between SOT groups; for example, evidence for the effectiveness of standard exercise training is needed in kidney and liver transplant candidates and recipients, whereas examining novel modes of exercise training is a logical next step in heart and lung transplant candidates and recipients.

Home-based programs were recognized as an important avenue for exercise delivery and research, especially in the late posttransplant phase. Benefits of home exercise in the posttransplant phase included the transition toward independence and maintaining a physically active lifestyle. Innovations in Telehealth or remote monitoring of patients either pre- or posttransplant were identified as potential avenues for research. Long-term benefits of exercise training were discussed as a gap in current research that could be addressed through various home or communitybased exercise delivery modes (e.g. fitness centers, webbased platforms and "tele-rehabilitation").

\section{Health-care economics}

Considering that public and private health funders are interested in survival, sustained improvement in QOL and the cost-effectiveness of exercise interventions, it was discussed that a broader scope of research in exercise should be adopted and the impact of rehabilitation beyond physical function and exercise capacity is essential to demonstrating the effectiveness of this intervention. Outcomes of choice included length of hospital stay as an impact of pretransplant rehabilitation, economic outcomes (cost of rehabilitation) and health-care utilization (including rehospitalization), sustained improvements in $\mathrm{QOL}$, allograft survival, risk of infection, risk of rejection, cardiovascular morbidity and mortality.

\section{Opportunities for research in exercise for SOT}

A number of existing and emerging opportunities for conducting research and improving clinical care in the area of exercise for SOT arose from the meeting discussions (see Figure 1). It was apparent that existing infrastructure, expertise and human resources at the current transplant centers that could be utilized to conduct research. There was also potential to utilize new technologies such as Telehealth to support exercise and physical activity interventions for long-term management, and to accommodate those living in areas remote from the rehabilitation centers. There was a strong need to disseminate the best available evidence for exercise training in pre- and posttransplant patients through the formulation of "expert guidelines." The dissemination of expert guidelines could improve the availability of evidence-based exercise programs for SOT candidates and recipients. The existence of current partners that could assist with improving research productivity in the area of exercise and physical activity, including academic institutions, transplant organizations and research 


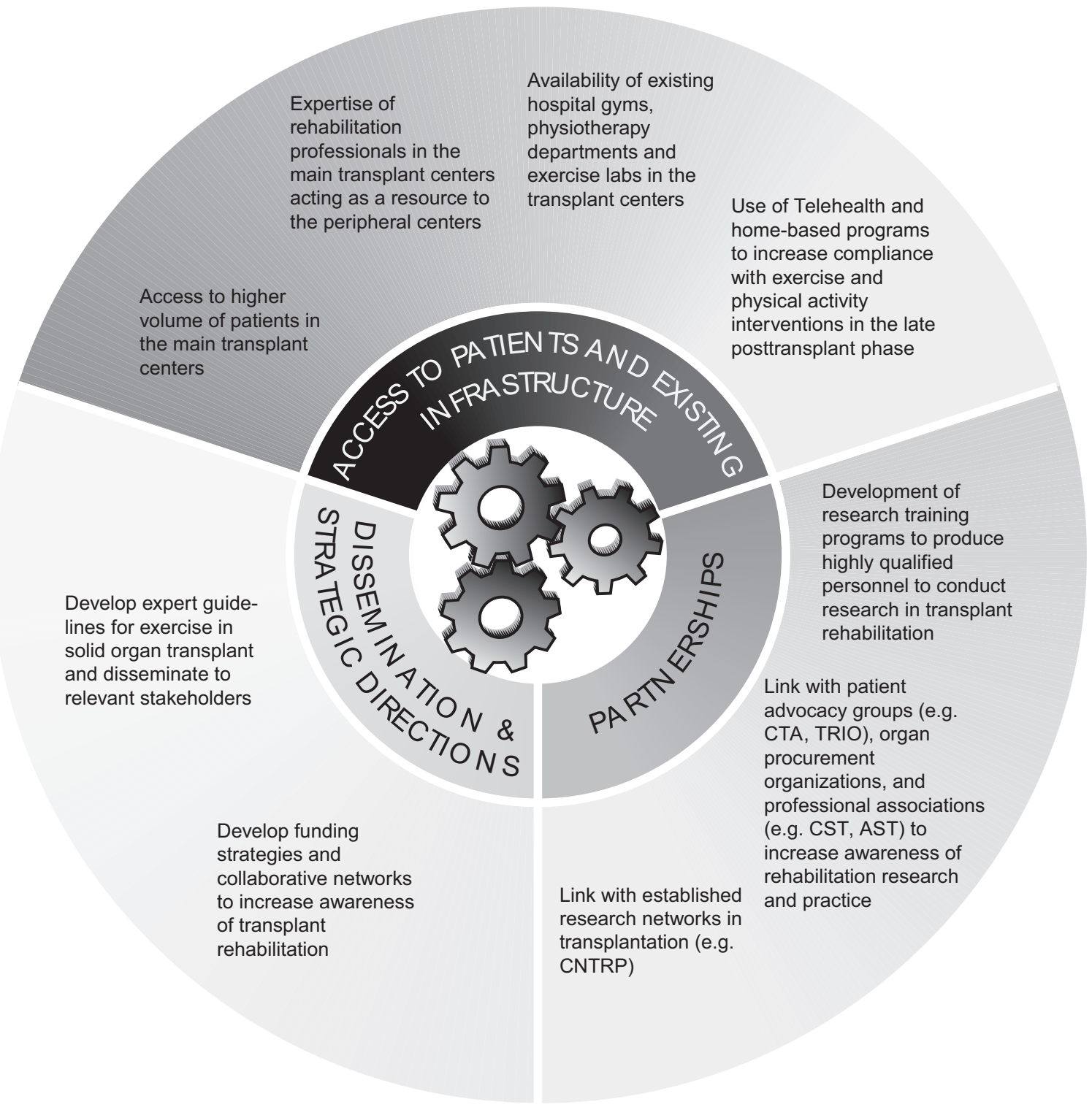

Figure 1: Existing and emerging opportunities to conduct research in rehabilitation for solid organ transplant recipients.

networks, were identified. The goal of developing partnerships and formalizing existing collaborations with these groups was emphasized as a way to move the research agenda forward.

\section{Summary}

Exercise and physical activity are important interventions that have the potential to improve outcomes for SOT candidates and recipients. To date, there is limited research supporting the benefits of exercise training, particularly for long-term benefits and outcomes beyond exercise capacity and $\mathrm{QOL}$. This meeting of experts and stakeholders in the area of exercise for SOT allowed for the development of top priorities for transplant research and a research agenda to streamline research efforts in this field to produce the most important and high quality evidence.

\section{Acknowledgments}

This meeting was supported by a Planning Grant from the Canadian Institutes of Health Research (ClHR) - Institute of Nutrition, Metabolism and Diabetes. The authors acknowledge the contributions of Ms. Margaret Duffy for facilitating the meeting, Ms. Lorna D'Souza for providing administrative 


\section{Mathur et al}

support and editorial assistance, and Dr. Erin Hamanishi for designing Figure 1. The authors thank Carman Hamilton, Pamela Hamilton and Sandra Holdsworth for sharing their personal experiences with transplant rehabilitation.

\section{Disclosure}

The authors of this manuscript have no conflicts of interest to disclose as described by the American Journal of Transplantation.

\section{References}

1. Scientific Registry of Transplant Recipients. Available at: http:// www.srtr.org/annual_Reports/2011/data_tables_section1.aspx. Accessed November 18, 2013

2. Zelle DM, Corpeleijn E, Stolk RP, et al. Low physical activity and risk of cardiovascular and all-cause mortality in renal transplant recipients. Clin J Am Soc Nephrol 2011; 6: 898-905.

3. Carey EJ, Steidley DE, Aqel BA, et al. Six-minute walk distance predicts mortality in liver transplant candidates. Liver Transpl 2010; 16: 1373-1378.

4. Lederer DJ, Arcasoy SM, Wilt JS, D'Ovidio F, Sonett JR, Kawut SM. Six-minute-walk distance predicts waiting list survival in idiopathic pulmonary fibrosis. Am J Respir Crit Care Med 2006; 174: 659-664.

5. Li M, Mathur S, Chowdhury NA, Helm D, Singer LG. Pulmonary rehabilitation in lung transplant candidates. J Heart Lung Transplant 2013; 32: 626-632.

6. Prentis JM, Manas DM, Trenell MI, Hudson M, Jones DJ, Snowden CP. Submaximal cardiopulmonary exercise testing predicts 90-day survival after liver transplantation. Liver Transpl 2011; 18: 152-159.

7. Kutner NG, Zhang R, Bowles T, Painter P. Pretransplant physical functioning and kidney patients' risk for posttransplantation hospitalization/death: Evidence from a national cohort. Clin J Am Soc Nephrol 2006; 1: 837-843

8. Didsbury M, McGee RG, Tong A, et al. Exercise training in solid organ transplant recipients: A systematic review and metaanalysis. Transplantation 2013; 95: 679-687.

9. Langer D, Burtin $C$, Schepers $L$, et al. Exercise training after lung transplantation improves participation in daily activity: $A$ randomized controlled trial. Am J Transplant 2012; 12: 15841592.

10. Krasnoff JB, Vintro AQ, Ascher NL, et al. A randomized trial of exercise and dietary counseling after liver transplantation. Am J Transplant 2006; 6: 1896-1905.

11. Williams TJ, McKenna MJ. Exercise limitation following transplantation. Compr Physiol 2012; 2: 1937-1979.

12. Kempeneers G, Noakes TD, van Zyl-Smit R, et al. Skeletal muscle limits the exercise tolerance of renal transplant recipients: Effects of a graded exercise training program. Am J Kidney Dis 1990; 16: 57-65.

13. Guerrero K, Wuyam B, Mezin P, et al. Functional coupling of adenine nucleotide translocase and mitochondrial creatine kinase is enhanced after exercise training in lung transplant skeletal muscle. Am J Physiol Regul Integr Comp Physiol 2005; 289: R1144-R1154.

14. Vivodtzev I, Pison C, Guerrero K, et al. Benefits of home-based endurance training in lung transplant recipients. Respir Physiol Neurobiol 2011: 177: 189-198.
15. Patcai JT, Disotto-Monastero MP, Gomez M, Adcock LE. Inpatient rehabilitation outcomes in solid organ transplantation: Results of a unique partnership between the rehabilitation hospital and the multi-organ transplant unit in an acute hospital. Open $\mathrm{J}$ Ther Rehabil. 2013; 1: 52-61.

16. Schakman O, Kalista S, Barbe C, Loumaye A, Thissen JP Glucocorticoid-induced skeletal muscle atrophy. Int J Biochem Cell Biol 2013; 45: 2163-2172.

17. Sakuma K, Yamaguchi A. The functional role of calcineurin in hypertrophy, regeneration, and disorders of skeletal muscle. J Biomed Biotechnol 2010; 2010: 721219.

18. Thomas RJ, King M, Lui K, Oldridge N, Pina IL, Spertus J. AACVPR/ACCF/AHA 2010. Update: performance measures on cardiac rehabilitation for referral to cardiac rehabilitation/ secondary prevention services endorsed by the American College of Chest Physicians, the American College of Sports Medicine, the American Physical Therapy Association, the Canadian Association of Cardiac Rehabilitation, the Clinical Exercise Physiology Association, the European Association for Cardiovascular Prevention and Rehabilitation, the Inter-American Heart Foundation, the National Association of Clinical Nurse Specialists, the Preventive Cardiovascular Nurses Association, and the Society of Thoracic Surgeons. J Am Coll Cardiol 2010; 56: 1159-1167.

19. Spruit MA, Singh SJ, Garvey C, et al. An official American Thoracic Society/European Respiratory Society statement: Key concepts and advances in pulmonary rehabilitation. Am J Respir Crit Care Med 2013; 188: e13-e64.

20. Dorn J, Naughton J, Imamura D, Trevisan M. Results of a multicenter randomized clinical trial of exercise and long-term survival in myocardial infarction patients: The National Exercise and Heart Disease Project (NEHDP). Circulation 1999; 100: 17641769.

21. Painter $\mathrm{PL}$, Hector $\mathrm{L}$, Ray $\mathrm{K}$, et al. $\mathrm{A}$ randomized trial of exercise training after renal transplantation. Transplantation 2002; 74: 42 48

22. Bernardi L, Radaelli A, Passino C, et al. Effects of physical training on cardiovascular control after heart transplantation. Int $\mathrm{J}$ Cardiol 2007; 118: 356-362.

23. Hermann TS, Dall CH, Christensen SB, Goetze JP, Prescott E, Gustafsson F. Effect of high intensity exercise on peak oxygen uptake and endothelial function in long-term heart transplant recipients. Am J Transplant 2011; 11: 536-541.

24. Tegtbur U, Pethig K, Machold $H$, Haverich A, Busse M. Functional endurance capacity and exercise training in long-term treatment after heart transplantation. Cardiology 2003; 99: 171176.

25. Braith RW, Mills RM, Welsch MA, Keller JW, Pollock ML. Resistance exercise training restores bone mineral density in heart transplant recipients. J Am Coll Cardiol 1996; 28: 14711477.

26. Mitchell MJ, Baz MA, Fulton MN, Lisor CF, Braith RW. Resistance training prevents vertebral osteoporosis in lung transplant recipients. Transplantation 2003; 76: 557562 .

27. Christensen SB, Dall CH, Prescott E, Pedersen SS, Gustafsson F. A high-intensity exercise program improves exercise capacity, self-perceived health, anxiety and depression in heart transplant recipients: A randomized, controlled trial. J Heart Lung Transplant 2012: 31: 106-107.

28. Wu YT, Chien CL, Chou NK, Wang SS, Lai JS, Wu YW. Efficacy of a home-based exercise program for orthotopic heart transplant recipients. Cardiology 2008; 111: 87-93. 
Exercise in Solid Organ Transplantation

29. Ihle F, Neurohr C, Huppmann P, et al. Effect of inpatient rehabilitation on quality of life and exercise capacity in long-term lung transplant survivors: A prospective, randomized study. J Heart Lung Transplant 2011; 30: 912-919.

30. Nytroen K, Rustad LA, Aukrust P, et al. High-intensity interval training improves peak oxygen uptake and muscular exercise capacity in heart transplant recipients. Am J Transplant 2012; 12 : 3134-3142.

\section{Supporting Information}

Additional Supporting Information may be found in the online version of this article.

Meeting Agenda: Invited Speaker Presentations

Facilitated Discussions 